Communication

\title{
Can a Dynamo Mechanism Act at the Magnetopauses of Magnetic Rapidly Rotating Exoplanets?
}

\author{
Elena Belenkaya (D)
}

Citation: Belenkaya, E. Can a Dynamo Mechanism Act at the Magnetopauses of Magnetic Rapidly Rotating Exoplanets? Fluids 2022, 7 , 60. https://doi.org/10.3390/ fluids7020060

Academic Editor: John Shebalin

Received: 18 November 2021

Accepted: 28 January 2022

Published: 31 January 2022

Publisher's Note: MDPI stays neutral with regard to jurisdictional claims in published maps and institutional affiliations.

Copyright: (C) 2022 by the author. Licensee MDPI, Basel, Switzerland. This article is an open access article distributed under the terms and conditions of the Creative Commons Attribution (CC BY) license (https:// creativecommons.org/licenses/by/ $4.0 /)$.
Federal State Budget Educational Institution of Higher Education M.V., Lomonosov Moscow State University, Skobeltsyn Institute of Nuclear Physics (SINP MSU), 119991 Moscow, Russia; elena@dec1.sinp.msu.ru

\begin{abstract}
An astrophysical dynamo converts the kinetic energy of fluids into magnetic energy. Dynamo is a non-local process. Here, we consider whether a dynamo can operate at the magnetopauses of magnetic rapidly rotating planets. We analyze the main necessary condition for the work of this type of dynamo- the rotation transfer from the planet to the magnetopause. We show the role of the current disc around a rapidly rotating magnetic planet in the redistribution of angular momentum depending on the direction of the external magnetic field, using the example of the Jupiter's magnetodisc.
\end{abstract}

Keywords: dynamo; Jupiter's magnetodisc; magnetosphere; angular momentum; magnetopause

\section{Introduction}

Two non-local processes are very important for space plasma physics: reconnection and dynamo. Magnetic reconnection, for example, Ref. [1] converts magnetic energy into kinetic energy, heat, and energy of accelerated particles. This occurs in a relatively small diffusion region, but its effect is manifested on a large scale. The dynamo, e.g., [2] vice versa, converts the kinetic energy of electrically conducting fluids into magnetic energy. This process also takes place in a small diffusion region, but the generated amplified magnetic field exists on a large spatial scale. To sustain these processes, energy sources are required. Here, we pay attention to the last of these mechanisms and to its energy source.

The sources of energy for plasma flows that generate dynamo can be different. Here, we are interested in a dynamo generated by the braking of rotation (this process can be considered as differential rotation) at the boundaries of magnetospheres of magnetic fast-spinning planets. The magnetosphere is a cavity around the planet, controlled by its magnetic field and plasma. The magnetospheric magnetic field includes not only the planetary field but also the field of magnetospheric current systems. The magnetospheric plasma consists of the ionospheric/exospheric plasma and may include some parts of the stellar wind plasma and the plasma from some volcanic moons if they exist. The magnetospheric boundary is called magnetopause.

For the dynamo to work, axial and mirror symmetry must be violated. Mirror symmetry breaking means the predominance of vortices of the same sign, i.e., the presence of average helicity. Mirror asymmetric random movements can generate large-scale magnetic fields. The Coriolis force reduces the geometric symmetry of the system. In addition, the existence of a diffusion zone with decreased conductivity is necessary for dynamo action.

The transfer of angular momentum can be carried out, for example, by the magnetorotational instability or MRI. The magnetorotational instability was described, for example, by Balbus and Hawley [3]. They showed how MHD turbulence arises in the accretion discs in the presence of a weak magnetic field and outwardly decreasing differential rotation. This leads to an increase in the effective viscosity and magnetic field generation. Masada et al. [4] investigated the non-linear properties of MRI using the three-dimensional numerical simulations of compressible MHD for core-collapse supernovae. The authors 
found that for proto-neutron stars with a milliseconds period and a magnetic field of $10^{5} \mathrm{G}$, the energy dissipation rate can exceed $10^{51} \mathrm{erg} \mathrm{s}^{-1}$. Reboul-Salze et al. [5] considered the efficiency of magnetorotational instability in the generation of a strong magnetic field in magnetars. The authors obtained a turbulent MRI-driven dynamo with a magnetic field in the proto-neutron stars $>2 \times 10^{15} \mathrm{G}$, which confirms the idea that magnetorotational instability can generate a strong magnetic field of magnetar. Probably, this mechanism can also work at the magnetopause of a rapidly rotating magnetic planet. However, for the angular momentum transfer from the planet to magnetopause inside the magnetosphere with collisionless plasma and highly conducting magnetic field lines, we assume that the main agent is the electric corotation field.

The magnetic field plays a significant role in the transfer of rotation from celestial bodies to great distances, as the plasma motion is determined by the interaction of the external magnetic field with the magnetic field of the magnetosphere. We analyze the influence of the interstellar magnetic field on this process and emphasize the role of the current disc, which can arise around the magnetic rapidly rotating planet. We will show how the rotation transfer depends on the value of the Alfven radius along the path of the rotating plasma. Thus, we consider how plasma flows can amplify and modify a weak background poloidal magnetic field just outside the magnetopauses of magnetized fast-rotating planets using Jupiter as an example.

\section{Dynamo Generated by Rotation Braking at the Magnetopause of Jupiter}

In the Jovian magnetosphere, due to the strong planetary magnetic field, fast rotation, and the presence of an additional plasma source-the volcanic moon Io-a current disc, called a magnetodisc, is formed around the planet. The transfer of rotation from the planet to the outer regions has its own peculiarities in the Jovian magnetosphere. A thin shell of strong field-aligned currents associated with the main auroral ovals arises at about $\sim 20 R_{\mathrm{J}}$, where $R_{\mathrm{J}} \sim 7 \times 10^{7} \mathrm{M}$ is Jupiter's radius [6-11]. It is assumed that there are no distributed field-aligned currents closer to the planet from this field-aligned current shell and, correspondingly, there is no azimuthal magnetic field $B_{\varphi}$ there. Behind these fieldaligned currents flowing out from the ionosphere, reverse field-aligned currents, closed in the ionosphere and in the current disc, can be distributed or concentrated.

Different rotating celestial bodies transfer rotation to different distances. For example, in the terrestrial magnetosphere, the corotation of plasma stops at the plasmapause located at $\sim 4-5 R_{\mathrm{E}}$ from the center of the planet in the equatorial magnetospheric plane, where $R_{\mathrm{E}} \sim 6.4 \times 10^{6} \mathrm{M}$ is the Earth radius. In the case of Jupiter, the rotation can be transferred to the magnetopause by the electric corotation field.

Thus, in the presence of a strong magnetic field of a fast-rotating planet, the rotation can be transferred to large distances (for a low but sufficient amount of magnetospheric plasma) up to the surrounding interplanetary medium, where it is slowing down. There, the energy of rotation, in principle, can be converted into magnetic energy if, at least, the necessary conditions for the dynamo action are carried out.

Jupiter has the strongest intrinsic magnetic field of the planets in the solar system, which defines the largest magnetosphere. In the 1950s, from the observed decametric and decimetric radio emission, it was found that Jupiter has its own magnetic field. Later, Pioneer 10 and 11 in 1973 and 1974 measured the Jovian magnetic field. The dipole magnetic moment of Jupiter $M_{\mathrm{J}} \sim 4.2 \times 10^{5} \mathrm{nT}_{\mathrm{J}}{ }^{3}$ is directed northward. Its angle with the rotation axis is $\sim 10^{\circ}[9,12]$. The Jovian rotation period is $\sim 10 \mathrm{~h}$ [9]. Jupiter's atmosphere transfers torque from the planet to the ionosphere, where the plasma rotates due to ion collisions with neutrals [6]. The electric corotation field $\boldsymbol{E}_{\text {cor }}$ is transmitted into the magnetosphere along highly conducting magnetic field lines:

$$
E_{\mathrm{cor}}=-\left[\Omega_{\mathrm{J}} \times r\right] \times B,
$$

(in the unmoving frame of reference), where $B$ is the magnetic field, $r$ is the jovicentric radius-vector, and $\Omega_{\mathrm{J}} \sim 1.76 \times 10^{-4} \mathrm{~s}^{-1}$ is the angular velocity of Jupiter. Corotation of 
magnetospheric plasma occurs under the action of $E_{\text {cor }}$. Electric potential corresponding to rigid corotation in the dipole magnetic field is:

$$
U_{\text {rig }}=\left(B_{0 \mathrm{~J}} \Omega_{\mathrm{J}} R_{\mathrm{J}}^{3} / \mathrm{r}\right) \sin ^{2} \theta,
$$

where $B_{0 \mathrm{~J}}$ is the magnetic field at the Jovian equator, and $\theta$ is a polar angle measured from the dipole magnetic field axis (co-latitude). Magnetospheric magnetic field lines are highly conductive, so in zero approximation, it is assumed that they are equipotential and transfer the $U_{\text {rig }}$ into the magnetosphere. The rigid corotation velocity is:

$$
\mathbf{V}_{\text {rig }}=r \Omega_{\mathrm{J}} \sin \theta \boldsymbol{e}_{\varphi}
$$

where $\boldsymbol{e}_{\varphi}$ is the unit vector in the azimuth direction.

In fact, the Jovian magnetospheric magnetic field includes not only a planetary dipole (and terms of a higher order) but also magnetic fields from other magnetospheric current systems: magnetopause shielding currents, magnetodisc, and tail current systems. All these sources of the magnetic field are described in the paraboloid model of Jupiter's magnetosphere, in which the magnetopause is approximated by a paraboloid of revolution, for example [13,14]. Distance from the center of the planet to the magnetopause subsolar point is $R_{\mathrm{ss}}$. The solar wind and its magnetic field from Jupiter's magnetosphere continuously interact with it. If the interplanetary magnetic field (IMF) is zero, the magnetosphere is closed: the magnetic field lines do not intersect the magnetopause. In this case, all magnetospheric field lines are divided into two classes: closed and open through the tail. Closed field lines have both footprints on the planet, while open lines are stretched into the tail and have one footprint on the planet and the other end in the solar wind. These open field lines transfer the rotation to the distant tail, where it gradually damps. Belenkaya [13], using the paraboloid model of the Jovian magnetosphere by Alexeev and Belenkaya [14], showed that the footprints of open field lines, going in the tail, have magnetic latitudes from $\sim 82-84^{\circ}$ to $90^{\circ}$ (for the chosen model parameters). Thus, in this case, the rotation is not transmitted to the magnetopause but remains locked in the magnetosphere.

For an IMF antiparallel to the planetary dipole moment (southward for the case of Jupiter, which magnetic moment is northward), the open field line flux turns from the distant tail to the equatorial plane of the magnetosphere, through which it goes into the opposite hemispace. In this case, in the equatorial magnetospheric plane, we obtain a system of two vortices: the nearest one surrounds the planet and corotates with it. It is located on closed field lines, while the distant vortex of anticorotation is placed on open field lines.

In the equatorial magnetosphere, corotation of magnetospheric plasma begins to break down beyond the Alfven radius - the distance from the planetary center at which the density of kinetic energy equals the density of magnetic energy. In Jupiter's equatorial magnetosphere, the Alfven radius is placed approximately at $\sim 20 R_{\mathrm{J}}$ (somewhere between Ganymede and Callisto [15]). Under the action of centrifugal force, the magnetospheric plasma with a significant fraction of iogenic ions begins to move radially outward in the equatorial magnetosphere beyond the Alfven radius, which leads to a decrease in the azimuthal velocity there. Since the open field line flux intersects the equatorial plane much farther from the Alfven radius, rotation on it should be practically absent and not be transferred to the magnetopause. Thus, the presence of a magnetodisc, on which rigid corotation slows down, prevents the rotation transfer to the magnetopause.

For an IMF almost parallel to the planetary dipole moment (northward for the case of Jupiter), open field lines do not intersect the equatorial magnetospheric plane, except the single one-separatrix - dividing open, closed, and interplanetary field lines. In this case, all open field lines intersect the corresponding magnetopause, and there is no significant rotation decrease on them since they do not cross the magnetodisc and equatorial region beyond it. The Alfven radius along open field lines, in this case, is much larger than in the 
low-latitude magnetosphere. At the Alfven radius, the flow velocity is equal to the Alfven velocity $V_{\mathrm{A}}$ (according to definition). The Alfven velocity is:

$$
V_{\mathrm{A}}=B /\left(\mu_{0} \cdot n_{\mathrm{i}} \cdot m_{\mathrm{i}}\right)^{1 / 2}
$$

where $\mu_{0}$ is the permeability of the vacuum, $n_{\mathrm{i}}$ and $m_{\mathrm{i}}$ are the density and mass of ions, respectively. $V_{\mathrm{A}}$ in the Jovian tail lobes is very large $\sim 31 \times 10^{3} \mathrm{~km} \mathrm{~s}^{-1}$ [16,17], because the ion density there is extremely low $\sim 10^{-5} \mathrm{~cm}^{-3}$ [18] (much less than in the plasma sheet), and there are fewer heavy ions than in the equatorial region, which is rich in iogenic heavy ions. For these parameters, the Alfven radius in the tail lobes can be estimated as $2.5 \times 10^{3} R_{\mathrm{J}}[19]$, which exceeds the distance to the magnetopause. Thus, the northward IMF corotation can be directly transferred to the northern and southern magnetopause. For example, open field lines with footprints at latitudes $\geq 82^{\circ}$ transport rotation to the region at the magnetopause from its low-latitude dayside part through the cusp to the nightside rather distant tail part. We assume that corotation is stopped beyond the magnetopause.

A decrease in the rotation energy beyond the magnetospheric boundary can, under certain necessary conditions, lead to the generation of a magnetic field just out of the Jovian magnetopause due to the dynamo action [19]. In the work of Belenkaya [19], the main necessary conditions for a dynamo at the Jovian magnetopause are listed, and their fulfillment is verified. The nature of the dynamo is, in principle, three-dimensional, and this is fulfilled in the case of Jupiter. The background poloidal IMF, interacting with decreasing rotation outside the magnetopause (which can be considered as differential rotation), is stretched into a toroidal field. The Coriolis force (in the rotating frame of reference) twists the toroidal field in the meridional planes. The average density of kinetic helicity:

$$
h_{\mathrm{k}}=\langle\boldsymbol{V} \cdot \operatorname{curl} \boldsymbol{V}\rangle \neq 0
$$

arises out of the magnetopause.

In addition to the kinetic helicity density $\left(h_{\mathrm{k}}\right)$, in rotating fluids with MHD turbulence, there is a magnetic helicity density: $h_{\mathrm{M}}=\langle\boldsymbol{A} \cdot \boldsymbol{B}\rangle \neq 0$, where $\boldsymbol{B}=\operatorname{curl} \boldsymbol{A}$, and $\boldsymbol{A}$ is the vector potential of the magnetic field $\boldsymbol{B}$, for example [20]. $h_{\mathrm{M}}$ is a measure of the linkage and twist of the magnetic field lines. The magnetic helicity is important for the work of a large-scale dynamo. For example, Shebalin [21] presented theoretical analysis and computational results of the geodynamo generation by MHD turbulence. The model used was based on the connection between the dipole in the terrestrial core and the magnetic helicity in it. Shebalin [21] derived a value of $3 \mathrm{mT}$ for the Earth's average dipole magnetic field and stated that "without magnetic helicity there is no dipole field". Large-scale astrophysical magnetic fields arise in the interstellar medium, in stars and planets due to turbulent dynamo effects. Their formation may be associated with magnetic helicity, for example [22]. Teissier and Müller [23] have studied the magnetic helicity transferred from small to larger scales in the context of large-scale magnetic fields created by a magnetic dynamo.

For the work of dynamo, a dissipative zone is required. Belenkaya $[19,24]$ showed that the Jovian magnetopause plays the role of a dissipative zone. It was also shown that to enhance the magnetic field out of the magnetopause, it is necessary that the poloidal component of the velocity of plasma flux passing by the planet and the perpendicularity to the axis of the planetary rotation component of the magnetic field beyond the magnetopause should not be zero. This condition is fulfilled for the plasma flow and magnetic field of the solar wind passing by Jupiter's magnetosphere.

Following Belenkaya [19], we roughly estimate the possibility of extracting magnetic energy from the damping rotation kinetic energy. To increase the IMF outside the Jovian magnetopause, the rotation energy there must exceed the energy of the solar wind flow. This condition is valid for the distances $l$ from the planetary rotation axis, where $l_{0} \leq l \leq R_{\mathrm{ss}}$. Here $l_{0}=V_{0} / \Omega_{\mathrm{J}}$, and $V_{0}$ is the solar wind velocity. Based on Ulysses measurements at 5 AU during the 23rd solar cycle, the average values of the solar wind parameters are: $V_{0} \sim 400 \mathrm{~km} \mathrm{c}^{-1}, M_{\mathrm{A}} \sim 17$ (Alfven Mach number), and IMF $b_{0} \sim 0.7 \mathrm{nT}$ [25]. The mean 
value of the subsolar magnetopause distance from the planetary center was estimated as $R_{\mathrm{ss}} \sim 86 R_{\mathrm{J}}$ [25]. For Jupiter we receive $l_{0}=V_{0} / \Omega_{\mathrm{J}} \sim 32 R_{\mathrm{J}}$.

Moreover, at $l_{0} \leq l \leq R_{\mathrm{ss}}$, the dynamo mechanism can act when the kinetic energy density of damping rotation $\left(n_{\mathrm{ext}} m_{\mathrm{ext}}\left(\Omega_{\mathrm{J}} l\right)^{2} / 2\right)$ out of the magnetopause exceeds the magnetic energy density $\left(b^{2} / 2 \mu_{0}\right)$, which can be obtained there. Here $b$ is the generated magnetic field value in the solar wind and $m_{\mathrm{ext}}$, and $n_{\mathrm{ext}}$ is the mass and density of ions, respectively, outside the magnetopause. Thus,

$$
n_{\text {ext }} m_{\text {ext }}\left(\Omega_{\mathrm{J}} l\right)^{2} / 2 \geq b^{2} / 2 \mu_{0}
$$

This means that the rotation velocity $\left(\Omega_{\mathrm{J}} l\right)$ transferred to the magnetopause must be of the order of or greater than the Alfven velocity in the solar wind corresponding to the enhanced magnetic field, $b\left(V_{\text {Aext }}=b /\left(\mu_{0} m_{\mathrm{ext}} n_{\mathrm{ext}}\right)^{1 / 2}\right)$. From the assumption of the energy equipartition [26], we obtain from Formula (6):

$$
\left(\mu_{0} m_{\mathrm{ext}} n_{\mathrm{ext}}\right)^{1 / 2}=b /\left(\Omega_{\mathrm{J}} l\right)=b_{0} / V_{0 \mathrm{~A}}
$$

under the assumption that the plasma density in the dynamo region equals the undisturbed value. Here $V_{0 \mathrm{~A}}=b_{0} /\left(\mu_{0} m_{\mathrm{ext}} n_{\mathrm{ext}}\right)^{1 / 2}$ is the Alfven velocity of the undisturbed solar wind flow. From the definition $M_{\mathrm{A}}=V_{0} / V_{0 \mathrm{~A}}$ it follows: $V_{0 \mathrm{~A}}=V_{0} / M_{\mathrm{A}}=400 \mathrm{~km} \mathrm{~s}^{-1} / 17=23.5 \mathrm{~km} \mathrm{~s}^{-1}$. From Equation (7), we receive:

$$
b / b_{0} \cong \Omega_{\mathrm{J}} l / V_{0 \mathrm{~A}}
$$

Considering $l \geq l_{0}$, we can estimate the IMF enhancement outside the Jovian magnetopause relative to its undisturbed value as $b / b_{0} \geq 1.76 \times 10^{-4} \mathrm{~s}^{-1} 32 R_{\mathrm{J}} / 23.5 \mathrm{~km} \mathrm{~s}^{-1}=16.8$. Of course, this is a very rough estimate. Indeed, the plasma angular velocity at the magnetopause is less than the velocity of Jupiter's rigid rotation. The velocity decrease occurs in the ionosphere and magnetosphere. As mentioned above, for a favorable IMF orientation, along the open field lines, the Alfven radius is greater than the distance to the magnetopause; therefore, the magnetic energy density is higher than the kinetic energy density, and the plasma follows the magnetic field. So, the decrease in rotation velocity in the magnetosphere is not large for this case.

In addition, the energy of damping rotation outside the magnetopause is not completely transferred to the magnetic energy. So, we obtain the upper limit of magnetic field amplification by the described mechanism.

\section{Application to Exoplanets}

As applied to the study of fast-rotating magnetized exoplanets, this means that one of the necessary conditions for a dynamo is the presence of a poloidal velocity component in the plasma flow around the magnetosphere and the difference in the direction of the interstellar magnetic field from the directions of the planetary rotation and magnetic axes.

Moreover, as shown above, for the proposed mechanism, it is necessary that rotation is transferred to the magnetopause, which is relevant for magnetic rapidly rotating exoplanet only if the stellar wind magnetic field is almost parallel (but not strictly parallel) to the axis of the planet's magnetic dipole. In this case, rotation is directly transferred to the magnetopause if the plasma density in the lobes is rather low but sufficient to support rotation.

Another conclusion for exoplanets made from the study of Jupiter's magnetosphere is an estimate based on a comparison of the kinetic energy density of damped rotation and the magnetic energy density of the enhanced magnetic field $B^{2} / 2 \mu_{0}$ obtained from it. The first energy density should be greater than the second, but the kinetic energy density of rotation $n_{\mathrm{i}} \cdot m_{\mathrm{i}}(\Omega l)^{2} / 2$ depends on the distance from the planetary rotation axis $l$, which is limited by the distance to the front point of the magnetopause $R_{\mathrm{sS}}$, which is the magnetospheric size scale: $l \leq R_{\mathrm{ss}}$. $R_{\mathrm{ss}}$ is determined by the equality of pressures on both 
sides of the magnetopause: the total pressure of the stellar wind should be equal to the total magnetospheric pressure at the front point of the magnetopause.

For magnetic field amplification, another condition must be fulfilled: the rotation velocity $\Omega l$ must be greater than the stellar wind velocity $V_{\mathrm{sw}}$. Thus, magnetic field amplification out of the magnetopause is possible for $l>V_{\mathrm{sw}} / \Omega$, or the inverse Rossby number $R_{0}^{-1}$ must be greater than 1 :

$$
R_{0}{ }^{-1} \equiv \Omega l / V_{\mathrm{sw}}>>1
$$

From these two constraints, we obtain the size of the dynamo region outside the magnetopause at a distance $l$ from the rotation axis of the planet:

$$
V_{\mathrm{sw}} / \Omega<l \leq R_{\mathrm{sS}}+\delta
$$

where $\delta$ is the width of the layer just beyond the magnetopause, in which the circulation breaks down. For the planets of the solar system, only Jupiter with its giant magnetosphere satisfies this requirement [19].

\section{Conclusions}

The magnetic reconnection of the interplanetary and magnetospheric magnetic fields plays a significant role in the magnetospheric magnetic field structure and, therefore, for the angular momentum transfer along highly conducting open magnetic field lines. Thus, the interplanetary magnetic field, especially its direction relative to the direction of the planet's magnetic field, controls the rotation transport.

In this paper, the possibility of the magnetic field amplification outside the magnetopause of a rapidly rotating magnetic planet by the dynamo mechanism powered by the energy of braking rotation in the dynamo region is discussed. To implement this process, it is necessary to transfer the rotation to the magnetopause without significant damping.

Under certain conditions, the magnetodisc can be formed in the magnetosphere of a rapidly rotating magnetic planet. Using the example of Jupiter's magnetosphere, we show that the implementation of the rotation transfer depends on whether the corresponding open magnetic field lines intersect the planetary magnetodisc or not. Our analysis reveals that the current disc around the planet plays a significant role in the redistribution of angular momentum because due to the radial outflow in it, the azimuthal plasma velocity slows down, and the field lines intersected the disc cannot transmit rotation further. Those open field lines that do not intersect the disc or the equatorial magnetosphere beyond it can transfer rotation to the boundary of the magnetosphere, beyond which it is gradually damped down.

It is noted for the first time that if the planet's magnetic field and angular rotation velocity are sufficiently large and there is enough plasma in the magnetosphere to form a magnetodisc, the magnetospheric size can be so large that the linear rotation speed at the magnetopause can exceed the external flow and Alfven velocities, and if the other necessary conditions are met $[19,24]$, the dynamo can act out of the magnetopause on those parts of it, dependent on the interplanetary magnetic field direction, where open field lines come, bypassing the magnetodisc and equatorial magnetosphere beyond it.

Funding: This research received no external funding.

Acknowledgments: Work was supported by the Ministry of Science and Higher Education of the Russian Federation under the Grant 075-15-2021-949.

Conflicts of Interest: The author declares no conflict of interest.

\section{References}

1. Priest, E.; Forbes, T. Magnetic Reconnection: MHD Theory and Applications; Cambridge University Press: Cambridge, UK, 2000.

2. Moffat, H.K. Magnetic Field Generation in Electrically Conducting Fluids; Cambridge University Press: Cambridge, UK, 1978.

3. Balbus, S.A.; Hawley, J.F. Instability and turbulence in accretion disks. Rev. Modern Phys. 1998, 70, 1-53. [CrossRef] 
4. Masada, Y.; Takiwaki, T.; Kotake, K.; Sano, T. Local simulations of the magneto-rotational instability in core-collapse supernovae. Astrophys. J. 2012, 759, 2. [CrossRef]

5. Reboul-Salze, A.; Guilet, J.; Raynaud, R.; Bugli, M. A global model of the magnetorotational instability in protoneutron stars Astronomy 2021, 645, A109. [CrossRef]

6. Hill, T.W. Inertial limit on corotation. J. Geophys. Res. 1979, 84, 6554-6558. [CrossRef]

7. Hill, T.W. The Jovian auroral oval. J. Geophys. Res. 2001, 106, 8101-8107. [CrossRef]

8. Caudal, G. A self-consistent model of Jupiter's magnetodisc including the effects of centrifugal force and pressure. J. Geophys. Res. 1986, 91, 4201-4221. [CrossRef]

9. Cowley, S.W.H.; Bunce, E. Origin of the main auroral oval in Jupiter's coupled magnetosphere-ionosphere system. Planet. Space Sci. 2001, 49, 1067-1088. [CrossRef]

10. Nichols, J.D.; Cowley, S.W.H. Magnetosphere-ionosphere coupling currents in Jupiter's middle magnetosphere: Effect of magnetosphere-ionosphere decoupling by field-aligned auroral voltages. Ann. Geophys. 2005, 23, 799-808. [CrossRef]

11. Connerney, J.E.P.; Kotsiaros, S.; Oliversen, R.J.; Espley, J.R.; Joergensen, J.L.; Joergensen, P.S.; Levin, S.M. A new model of Jupiter's magnetic field from Juno's first nine orbits. Geophys. Res. Lett. 2018, 45, 2590-2596. [CrossRef]

12. Smith, E.J.; Gulkis, S. The magnetic field of Jupiter-A comparison of radio astronomy and spacecraft observations. Ann. Rev. Earth Planet. Sci. 1979, 7, 385-415. [CrossRef]

13. Belenkaya, E.S. The Jovian magnetospheric magnetic and electric fields: Effects of the interplanetary magnetic field. Planet. Space Sci. 2004, 52, 499-511. [CrossRef]

14. Alexeev, I.I.; Belenkaya, E.S. Modelling of the Jovian magnetosphere. Ann. Geophys. 2005, 23, 809-826. [CrossRef]

15. Belenkaya, E.S. Callisto in the magnetosphere of Jupiter. Sol. Syst. Res. 2020, 54, 99-109. [CrossRef]

16. Goldstein, M.L.; Lepping, R.P.; Sitter, E.C., Jr. Magnetic field properties of Jupiter's tail at distances from 80 to 7500 Jovian radii. J. Geophys. Res. 1985, 90, 8223-8239. [CrossRef]

17. Belenkaya, E.; Alexeev, I. Sliding contacts in planetary magnetospheres. Symmetry 2021, 13, 283. [CrossRef]

18. Gurnett, D.A.; Kurth, W.S.; Scarf, F.L. The structure of the Jovian magnetotail from plasma wave observations. Geophys. Res. Lett. 1980, 7, 53-56. [CrossRef]

19. Belenkaya, E.S. Generation of the magnetic field at the magnetopauses of the rapidly rotating planets. J. Geophys. Res. 1996, 101, 41-47. [CrossRef]

20. Lund, K.; Jardine, M.; Russell, A.J.B.; Donati, J.F.; Fares, R.; Folsom, C.P.; Jeffers, S.V.; Marsden, S.C.; Morin, J.; Petit, P.; et al. Field linkage and magnetic helicity density. Mon. Not. R. Astron. Soc. 2021, 502, 4903-4910. [CrossRef]

21. Shebalin, J.V. Magnetic Helicity and the Geodynamo. Fluids 2021, 6, 99. [CrossRef]

22. Müller, W.C.; Malapaka, S.K.; Busse, A. The inverse cascade of magnetic helicity in magnetohydrodynamic turbulence. Phys. Rev. E 2012, 85, 015302. [CrossRef]

23. Teissier, J.-M.; Müller, W.-C. Inverse transfer of magnetic helicity in supersonic magnetohydrodynamic turbulence. J. Phys. Conf. Ser. 2020, 1623, 012011. [CrossRef]

24. Belenkaya, E.S. Planetary magnetopause and heliopause current sheets. In Electric Currents in Geospase and Beyond; Keiling, A., Marghitu, O., Wheatland, M., Eds.; AGU Geophysical Monograph 235; John Wiley \& Sons, Inc.: Hoboken, NJ, USA, 2018; Chapter 13; pp. 207-217. ISBN 978-1-119-32449-2.

25. Ebert, R.W.; Bagenal, F.; McComas, D.J.; Fowler, C.M. A survey of solar wind conditions at 5 AU: A tool for interpreting solar wind-magnetosphere interactions at Jupiter. Front. Astron. Space Sci. 2014, 1, 13. [CrossRef]

26. Landau, L.D.; Lifshitz, E.M. Electrodynamics of Continuous; Media Pergamon: New York, NY, USA, 1959. 